\title{
EatRight Ontario (ERO): \\ A Dietitian Contact Centre and its impact on nutrition services
}

\author{
Christine Mehling', Helen Haresign', Honey Bloomberg', Elaine Wu', Cameron D. Norman ${ }^{3}$, Jeanne Legare² \\ 'EatRight Ontario, Dietitians of Canada, 'Jeanne Legare and Associates, ${ }^{3}$ Dalla Lana School Public Health University of Toronto
}

Introduction: ERO was a multi-modal dietitian contact centre, managed by Dietitians of Canada that offered free, evidence based healthy eating advice, based on PEN: Practice-based Evidence in Nutrition $®$ and behaviour change support to Ontarians. Access was by phone, email or website.

Objectives: To determine ERO's impact on consumer outcomes, health intermediaries $(\mathrm{HI})$ supports, and nutrition service delivery in Ontario.

Methods: An e-survey link was sent to consumers in the fall of 2017, I - 4 weeks after contacting ERO, to assess perceived benefits, satisfaction and self-reported outcomes. $48 \%$ of respondents provided email addresses after using the ERO phone service, while $52 \%$ followed a link to the survey through the ERO's consumer eNews Bulletin. Consumers were streamed based on inquiry; diabetes, infant/child feeding, and all other nutrition topics. Health intermediaries were e-surveyed to assess the impacts of ERO on their professional practice, agency and nutrition service delivery. $\mathrm{HI}$ were invited to participate through ERO's $\mathrm{HI}$ eNews bulletin.

Results: 867 consumers and $337 \mathrm{HI}$ completed the survey. Of consumers $\mathbf{9 0 - 9 7 \% ~ w e r e ~ s a t i s f i e d ~ w i t h ~ t h e ~ s e r v i c e ~ a n d ~ i n d i c a t e d ~}$ that they understood the information provided, felt better informed, got the advice they needed and were able to use the advice provided (Table I). $93 \%$ of consumer respondents made $2-4$ desirable behaviour changes following their contact with ERO, or had their current behaviours confirmed (Table 2).

$95 \%$ of $\mathrm{HI}$ indicated that ERO provided a valuable service and positive impact on their service (Table 3 ). ERO provided access to evidence-based resources and advice (80\%), increased access to dietitians $(79 \%)$, supported government policies $(55-72 \%$, sector dependent) decreased their need to develop additional resources, and answer client inquiries. HI strongly valued ERO's role in supporting their professional practice, clients, and the work of their organization with minimal concerns of duplication of roles. $35 \%$ of $\mathrm{HI}$ surveyed indicated that ERO was a needed service. Without ERO, they felt there would be gap in accessing dietitians (58\%), credible information (49\%) and ERO resources (37\%).

Conclusions: Consumers indicate that ERO is a highly valued service positively impacting and improving health knowledge and behavior change. HI strongly valued ERO's role in supporting their professional practice and building capacity within their sector, and therefore shifted functions and consumer supports to ERO, like resource development and consumer inquiries, so that they could focus on other agency priorities.

Significance to Dietetics: ERO, a multi-modal dietitian contact centre provided an innovative approach to access dietitians and engage consumers, which can serve as a prototype for others. Quantitative and qualitative data show that ERO played a trusted role in providing nutrition knowledge and behavior change to consumers. $\mathrm{HI}$ relied on this service, and indicated that its absence would create strategic and operational gaps in nutrition service delivery in Ontario.

Dietitians of Canada acknowledges the past financial support of EatRight Ontario by the Ontario government. The views expressed do not necessarily reflect those of the Province.
Table I

\begin{tabular}{|c|c|c|c|}
\hline \multicolumn{4}{|c|}{$\begin{array}{l}\text { Information Utilization by Inquiry Type } \\
\% \text { "strongly agree" or "agree" (consumer responses) }\end{array}$} \\
\hline Service Feature & $\begin{array}{l}\text { Diabetes } \\
\text { Inquiries } \\
(n=140)\end{array}$ & $\begin{array}{l}\text { Infant/Child } \\
\text { Inquiries } \\
(n=122)\end{array}$ & $\begin{array}{l}\text { All Other } \\
(n=522)\end{array}$ \\
\hline $\begin{array}{l}\text { I understand the } \\
\text { information provided }\end{array}$ & $97 \%$ & $96 \%$ & $95 \%$ \\
\hline I feel better informed & $93 \%$ & $95 \%$ & $92 \%$ \\
\hline $\begin{array}{l}\text { I got the advice I } \\
\text { needed }\end{array}$ & $92 \%$ & $94 \%$ & $90 \%$ \\
\hline $\begin{array}{l}\text { I am able to use the } \\
\text { advice given }\end{array}$ & $92 \%$ & $95 \%$ & $90 \%$ \\
\hline $\begin{array}{l}\text { I am satisfied with the } \\
\text { service }\end{array}$ & $92 \%$ & $94 \%$ & $92 \%$ \\
\hline
\end{tabular}

Table 2

\begin{tabular}{|l|l|l|}
\hline \multicolumn{3}{|l|}{ Top Behaviour Changes Reported by Consumers } \\
\hline $\begin{array}{l}\text { Diabetes } \\
(\mathbf{n = 1 4 3 )}\end{array}$ & $\begin{array}{l}\text { Infant/Child } \\
\text { Feeding } \\
\text { (n= I 23) }\end{array}$ & $\begin{array}{l}\text { Other } \\
(\mathbf{n = 5 5 6})\end{array}$ \\
\hline $\begin{array}{l}\text { Eat smaller portions } \\
(57 \%)\end{array}$ & $\begin{array}{l}\text { Offer child variety of } \\
\text { foods (42\%) }\end{array}$ & $\begin{array}{l}\text { Increase variety of } \\
\text { healthy foods (4I\%) }\end{array}$ \\
\hline $\begin{array}{l}\text { Eat more vegetables } \\
(54 \%)\end{array}$ & $\begin{array}{l}\text { Let child decide on how } \\
\text { much (33\%) }\end{array}$ & $\begin{array}{l}\text { Improve portion } \\
\text { control (34\%) }\end{array}$ \\
\hline $\begin{array}{l}\text { Choose healthier foods } \\
(53 \%)\end{array}$ & $\begin{array}{l}\text { Offer child iron rich } \\
\text { foods (28\%) }\end{array}$ & $\begin{array}{l}\text { Read food label } \\
(34 \%)\end{array}$ \\
\hline $\begin{array}{l}\text { Eat more fibre-rich } \\
\text { foods ((50\%) }\end{array}$ & $\begin{array}{l}\text { Offer child variety of } \\
\text { textures (22\%) }\end{array}$ & $\begin{array}{l}\text { Increase fibre-rich } \\
\text { foods (30\%) }\end{array}$ \\
\hline $\begin{array}{l}\text { Do more physical } \\
\text { activity (45\%) }\end{array}$ & $\begin{array}{l}\text { ERO confirmed what I } \\
\text { was doing (20\%) }\end{array}$ & $\begin{array}{l}\text { Decrease sugar intake } \\
(27 \%)\end{array}$ \\
\hline Median = 4 & Median = 2 & Median = 3 \\
\hline
\end{tabular}

\section{Table 3}

\begin{tabular}{|l|l|l|l|}
\hline \multicolumn{4}{|l|}{ Value of Service to Health Intermediaries } \\
\hline Statement about ERO & $\begin{array}{l}\text { All HI } \\
(\mathbf{n = 3 3 5})\end{array}$ & $\begin{array}{l}\text { Public } \\
\text { Health } \\
(\mathbf{n = 1 8 8})\end{array}$ & $\begin{array}{l}\text { RD/ } \\
\text { Nutrition } \\
(\mathbf{n}=\mathbf{1 7 8})\end{array}$ \\
\hline Excellent or good value & $90 \%$ & $95-99 \%$ & \\
\hline Trustworthy information & $100 \%$ & $100 \%$ & $100 \%$ \\
\hline $\begin{array}{l}\text { Compliments my } \\
\text { service }\end{array}$ & $96 \%$ & $100 \%$ & $100 \%$ \\
\hline $\begin{array}{l}\text { Offers service that } \\
\text { would not be provided } \\
\text { due to funding/scope }\end{array}$ & $71 \%$ & $94 \%$ & $83 \%$ \\
\hline Duplicates my service & $7 \%$ & $10 \%$ & $6 \%$ \\
\hline
\end{tabular}

